\title{
THE USE OF COMPUTER-GUIDED SURGERY VIA A PARTIALLY GUIDED PROTOCOL DOES NOT COMPLETELY COMPENSATE FOR THE LEVEL OF OPERATOR EXPERIENCE IN IMPLANT PLACEMENT
}

\author{
Hesham F. Marei *
}

\begin{abstract}
Purpose: There is always a tension between allowing novice trainees to place implants independently and the need to achieve accurate functional and esthetic outcomes. We aimed to measure the influence of surgeon experience on the accuracy of implants placed using a teethsupported surgical guide via a partially guided surgical protocol in a clinical setting.

Materials and Methods: Twenty partially edentulous patients were randomly allocated to expert and novice surgeon groups. Implant drilling and placement for the two groups were performed through a flapless surgical technique using surgical guides following a partially guided surgical protocol. The study primary independent variable was the surgeon experience, while the outcome variable was the accuracy, which was measured based on the differences in implant angulations pre- and postoperatively. Two-way analysis of variance (ANOVA) was applied to find the influence of surgeon experience, implant size, and the interactive effect of surgeon experience and implant size on the angular deviation.
\end{abstract}

Results: A total of 40 implants were inserted in 7 males and 13 females. There was no significant difference $(\mathrm{p}=0.453)$ in the mesio-distal deviation between the expert and novice groups. In a bucco-lingual direction, the expert group performed more accurate implant placement $(3.7 \pm 3.35)$ compared with the novice surgeons $(8.5 \pm 6.3)$. There was no effect for implant size and/or the interaction effect on the degree of angular deviations.

Conclusion: The level of surgeon experience affects the accuracy of implants placed using a teeth-supported surgical guide in a bucco-lingual direction; therefore, the use of computer-guided surgery via a partially guided protocol does not completely compensate for the level of operator experience. However, such surgical guide might be used in a whole task training of novice surgeons, as it can bridge a gap between simulation training in vitro and free-hand surgery in a clinical setting.

* Assistant Professor of Oral and Maxillofacial surgery, Faculty of Dentistry, Suez Canal University, Ismaillia, Egypt. 


\section{INTRODUCTION}

A surgical guide that is fabricated through computer-aided design and computer-aided manufacturing (CAD/CAM) or laboratory-based conventional procedures has been used in an attempt to increase the accuracy of implant placement. Computer-guided surgery enables flapless implant insertion, ${ }^{1}$ and reduces the surgical time, postoperative pain, and swelling. ${ }^{2}$ It can facilitate the immediate insertion of prefabricated fixed prostheses, which improves patient satisfaction. ${ }^{3}$ Furthermore, it can achieve higher precision and accuracy in cases of high esthetic demand and insufficient bone height. ${ }^{4}$ However, it involves an increase in planning time and additional costs relating to the planning software and the production of the stereolithographic surgical guide. ${ }^{1}$

In 2012, as a part of third European Association of Osseointegration (EAO) conference on computerguided surgery, a group of eminent members analyzed two systematic reviews and a metaanalysis on the accuracy of guided surgery. They concluded that "Guided surgery does not guarantee a precise implant placement." The authors reported a mean deviation of $1.09 \mathrm{~mm}$ at entry, $1.28 \mathrm{~mm}$ at the apex, and $3.9^{\circ}$ in angulation of implants inserted using guided surgery techniques. In the horizontal and vertical directions, the mean error was $1.2 \mathrm{~mm}$ and $0.5 \mathrm{~mm}$, respectively. ${ }^{1}$

Precision in implant placement, when a stereolithographic surgical guide is used, depends on multiple factors that lead to cumulative and interactive errors, from data-set acquisition to the surgical procedure. ${ }^{5}$ The first group of errors is incorporated during the fabrication of the stereolithographic surgical guide, such as errors in cone-beam computed tomography (CBCT) image acquisition, ${ }^{6,7}$ surface registrations of the $\mathrm{CBCT}$, and the dental record. ${ }^{8,9}$

The second group of errors is related to the type of guide support during surgery, which could be bone, mucosa, or tooth support. ${ }^{10}$ Multiple studies have confirmed that the magnitude of error increases when bone-supported guides are used, ${ }^{5,10}$ whereas a tooth-supported template for the treatment of single missing teeth results in greater accuracy of implant positioning than with mucosa-supported or bonesupported templates. ${ }^{2}$ The third group of errors emerges during surgery, which could be either fully guided or partially guided. The partially guided surgery involves one or more steps of freehand drilling and freehand implant insertion. More accurate results are achieved with the fully guided surgery approach. ${ }^{11,12}$

Finally, the 3rd EAO research group added one more factor that might affect the accuracy during surgery, which is the surgeon experience. It was mentioned that guided implant surgery is technically demanding, and the belief that "less training is needed" is far from accurate. Furthermore, they stated that the surgical skills and experience of the clinician using this surgical technique go above and beyond those necessary for providing regular implant surgery. ${ }^{1}$

Multiple in-vitro studies have showed the statistically significant effect of surgeon experience on the accuracy of implant-guided placement. More experienced surgeons have placed more implants accurately when a bone-supported stereolithographic surgical guide is used, as there was a statistically significant difference for the angular and horizontal error at the implant apex and platform. ${ }^{13}$ Similar results were obtained when different teeth support surgical guides were used, and it was found that experienced surgeons perform less error in the bucco-lingual implant angulation. The authors anticipated that the magnitude of deviation in accuracy of implant angulation would be higher if novice surgeons operated in a real clinical setting. ${ }^{14}$

The real clinical setting is a complex environment that might exert a cognitive load on novice learners, negatively affecting their performance. 
In undergraduate dental education and implant residency programs, there is always a balance between allowing trainees to operate independently and the need to achieve the intended functional and esthetic outcomes. Therefore, the use of a stereolithographic surgical guide might help trainees to increase the accuracy of implant placement in the clinical setting, which could provide an opportunity for using computer-guided implant surgery as a scaffold between implant simulation training on models in vitro and freehand surgery on patients.

The purpose of this study was to measure the influence of surgeon experience on the accuracy of implants placed using teeth supported surgical guides via partially guided surgical protocols in a real clinical setting. Our independent variable is the surgeon experience, whereas our dependent variables are the degree of mesio-distal and buccolingual deviations of the inserted implants from the planned placement. The investigators hypothesize that the expert surgeons should place implants more accurate than the novice surgeons.

\section{MATERIALS AND METHODS}

\section{Study design and sampling}

To address the research purpose, the investigators designed and implemented a randomized controlled study. The study population was composed of all patients presenting to the oral and maxillofacial surgery department for replacing missing teeth between 03/ 2016 and 12/ 2017. This study was approved by the ethical committee of the university and all participants signed an informed consent agreement (IRB number 2015-02-101).

In this study you have two samples to describe and define, patients and clinicians. The patients' sample size was calculated based on angular deviations, which are recorded in the previous invitro studies ${ }^{13,15}$ (2.19, 3.96, and 7.63 degrees) and expected standard deviation of 1.4 degrees. A sample size of 10 patients per group is needed to obtain a power of $80 \%$ with a confidence level of $95 \%$. The patients were randomized into two groups (10 patients/group).

The study involved the participation of 20 patients (7 males and 13 females). All the patients accepted the treatment plans and provided signed informed consent for the surgery and the photographs.

To be included in the study sample, patients had to be medically fit, have remaining teeth that can support the surgical guide, have missing teeth posterior to the canine tooth. The edentulous area should involve healed bone sites at least 3 months after extraction with no history of bone graft, have bone height of at least $8 \mathrm{~mm}$ from the alveolar crest to the nearest vital structure and bone width of at least $2 \mathrm{~mm}$ beyond the intended implant diameter. Patients were excluded as study subjects if they are fully edentulous, pregnant, tobacco smokers of more than 10 cigarettes per day, if the edentulous sites require hard or soft tissue augmentation at the time of implant placement.

In regard to the clinicians, 4 clinicians (2 experts and 2 novices) were participated in the study. The novice surgeons are those who had completed theoretical training on dental implants and participated as observers only in previous implant surgical procedures on real patients. The expert surgeons had independently placed more than 100 implants through guided and conventional approaches over the past 2 years.

\section{Study variables}

The main independent (predictor) variable is surgeon experience (expert vs. novice). Moreover, the study has considered the size of the implant as a second independent variable as it affects the number of osteotomies required before implant insertion, which might affect the accuracy. The primary outcome variable was the accuracy of implant placement, which is calculated based on 
the difference in the angular deviation of the real implants from the virtual one. Implant placement is considered accurate if the angular deviation is less than $7.6+/-1.4$, which was the maximum reported deviation in an in vitro study.

\section{Preoperative planning}

The two groups of patients received the same surgical intervention by two different groups of surgeons. Group I received implants by two expert oral surgeons using a teeth support stereolithographic surgical guide. Group II received implants by two novice oral surgeons using the same type of surgical guide (teeth support stereolithographic surgical guide). All patients $(\mathrm{N}=20)$ were examined by an expert surgeon and expert prosthodontist, who formulated the implant treatment plan. Optical scanning was performed on patient preoperative study models (with and without wax pattern) to make two 3-dimensional (3D) digital models per patient. Images (STL file) were imported and uploaded with the patient preoperative CBCT DICOM images (Kodak CS 9300, Kodak Dental Systems, Carestream Health, Rochester, NY, USA) that were previously taken at $85 \mathrm{kV}$ and $6 \mathrm{~mA}$, voxel size $250 \mu \mathrm{m}$, to SIMPLANT pro $17 \AA$ software (Dentsply Implants NV, Kessel-Lo, Belgium). The images of the model were registered on the preoperative DICOM images based on a three-point surface registration technique that is offered by the planning software.

One expert surgeon planned all the implants $(\mathrm{N}=$ 40) in the most optimal position based on the available bone, vital structures, and the prosthetic demands (scanned wax pattern). For all patients $(\mathrm{N}=20)$ with guided surgery, the planning was transferred to the manufacturer (Dentsply Implants NV, Kessel-Lo, Belgium) for the fabrication of a stereolithographic drill guide. The implant parameters (mesial-distal and bucco-lingual angulations) were retrieved from the Simplant software. These measurements correspond to the virtual planning.

\section{Operative and post-operative protocol}

Implant drilling and placement for groups I and II were performed under local anesthesia through a flapless surgical technique. During surgery, the stability of the surgical guide was checked and confirmed by an expert surgeon. Extra transmucosal fixation of the guide using $1.5-\mathrm{mm}$ bone screws was performed in cases of posterior free end saddles (Figures 1-4).

For groups I and II, the drilling procedure involved the use of a universal surgical Simplant Kit (SIMPLANT, Dentsply Implants NV, KesselLo, Belgium). It involved drill keys to be inserted in the sleeves within the surgical guide, to guide the consecutive drills with different diameters in the correct positions and planned angulation. Different keys (2.2 mm, $2.8 \mathrm{~mm}$, and $3.2 \mathrm{~mm}$ ) with increasing diameters are available to guide each separate drill. The drilling depth corresponds to the planned implant length and the height of the sleeves. Therefore, the drilling has to go to the full drill length to achieve the desired implant length. All the drilling steps stop at a drill $3.2 \mathrm{~mm}$ in diameter; then, the osteotomy is finalized by the corresponding implant system drills (Zimmer Dental, Inc., USA).

The surgical guide is removed to finalize the osteotomy in freehand. Freehand drilling involves one drill $(3.4 \mathrm{~mm})$ to place a $3.7-\mathrm{mm}$ implant with a regular platform of $3.5 \mathrm{~mm}$, two drills (3.4 and $3.8 \mathrm{~mm}$ ) to place $4.1-\mathrm{mm}$ implants with a regular platform of $3.5 \mathrm{~mm}$, and three drills $(3.4,3.8$, and $4.4 \mathrm{~mm}$ ) to place $4.7-\mathrm{mm}$ implants with a wide platform of $4.5 \mathrm{~mm}$. Physical stoppers were present on all drills when the osteotomy was finalized to control the drilling depth. All implants (Tapered Screw Vent MTX, Zimmer Dental, Inc., USA) were inserted using the corresponding surgical kit. Implant insertion into the osteotomy for the two groups was performed in the freehand technique.

One week after implant placement, a CBCT scan (Kodak CS 9300, Carestream Health, USA) 
was taken using the same parameters to check the final position of the implants. The postoperative DICOM images were imported to SIMPLANT software. Using the SIMPLANT library, implants were selected and placed virtually over the real implants that appear in the DICOM images. Images were checked in an axial, coronal, sagittal, and 3D plans to ensure complete surface registration of the virtually placed implants and the real inserted implants.

\section{Data collection}

Using the implant properties option in the software, the mesio-distal and bucco-lingual angle measurements of the virtually placed implants were retrieved (Figures 5 and 6). Because there is a complete surface overlap of images, these measurements correspond to the real implant parameters (mesio-distal and bucco-lingual angulation of the real inserted implant). After 1 week, another investigator, who was blinded to the intervention, checked the accuracy of image overlap and made any necessary changes; then, the

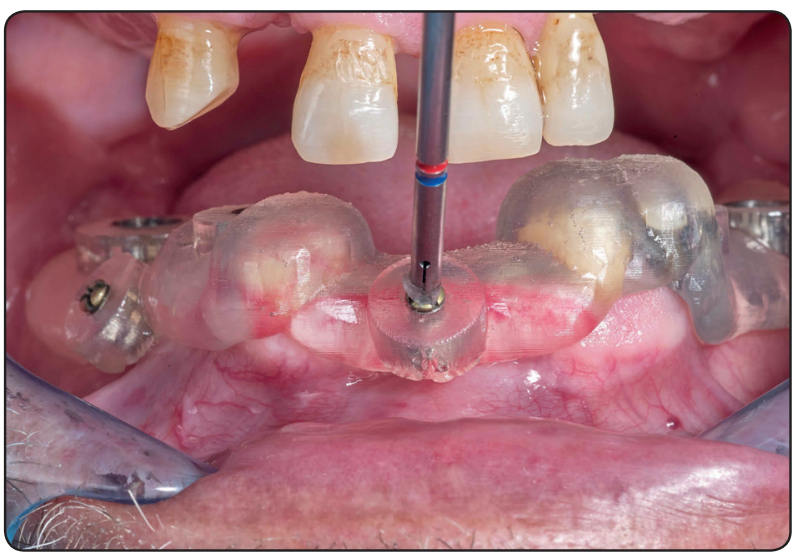

Fig. (1) Trans-mucosal fixation of the teeth supported surgical guide using $1.5-\mathrm{mm}$ bone screws in a free end saddle case. parameters of the real inserted implants (mesiodistal and bucco-lingual angulation) were retrieved for the second time. There was a significant positive correlation between the two raters. Therefore, the mean angulation of the first and second measurements was considered to be the final measurement.

\section{Data analysis}

All measurements were collected and tabulated using Microsoft Excel; then, a mathematical calculation was performed to find the angular deviation of the inserted implants from the planned one for all the groups. Two-way analysis of variance (ANOVA) test was applied using IBM SPSS statistics (version 22) to determine the influence of surgeon experience, implant size, and the interaction effect of surgeon experience and implant size on the angular deviation. To determine the inter-examiner variability, the Pearson correlation coefficient (-1: perfect negative correlation, 1: perfect positive correlation) was calculated for the angular deviation.

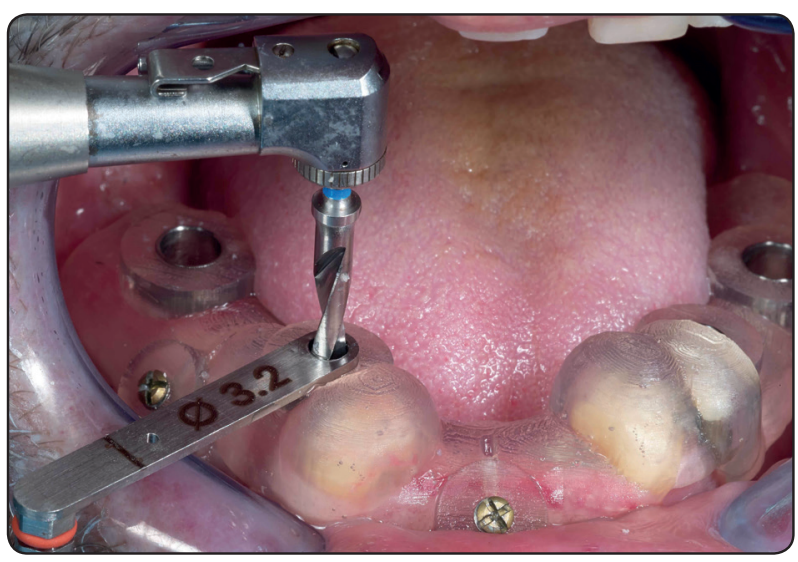

Fig. (2) Drilling using Simplant Universal Kit while a $3.2 \mathrm{~mm}$ in diameter drill key is inserted inside the metal sleeve at site 44 . 


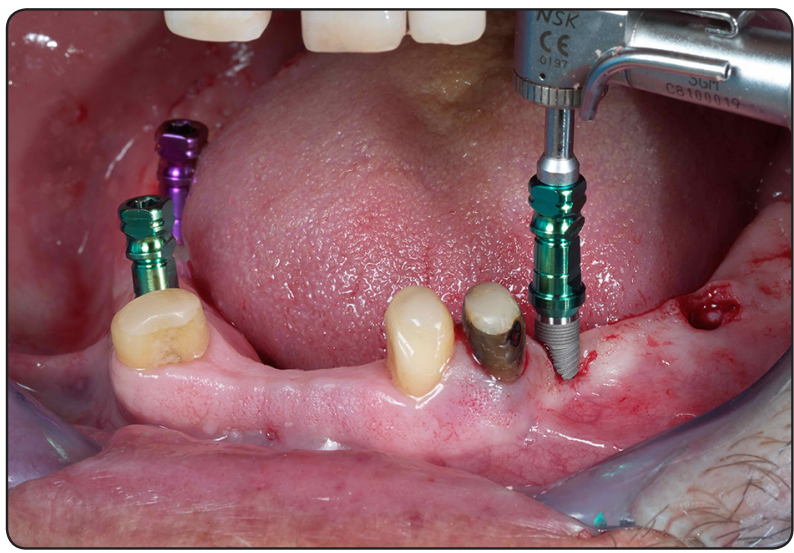

Fig. (3) Free hand implant insertion at site 35.

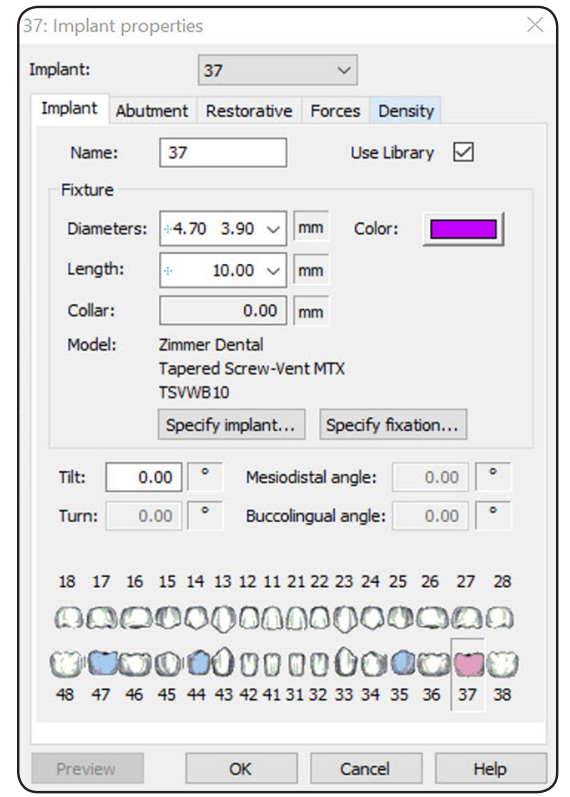

Fig. (5) Using implant property tool in Simplant software to calculate the Bucco-lingual and mesio-distal angles of the virtual plan of implant at site 37

\section{RESULTS}

A total of 20 patients ( 13 women 7 men, mean age $=47.1 \pm 10.3$ years) participated in our study, and received a total of 40 implants. Group I (5 females and 5 males) received 25 implants, and group II (8 females and 2 males) received 15 implants. All patients successfully received the implants, and there was no peri-implant infection among the patients. Patients reported pain and minimal swelling that lasted for 3 to 5 days after the surgery.

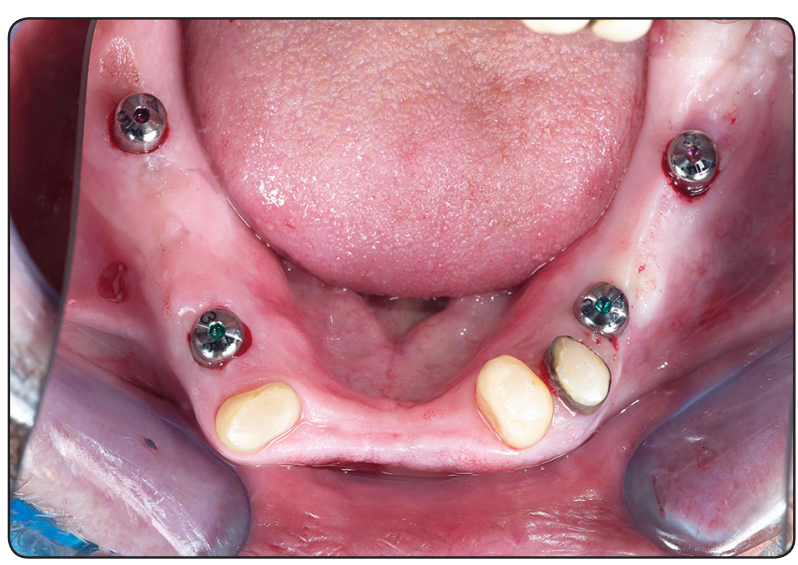

Fig. (4) Healing abutments are immediately placed.

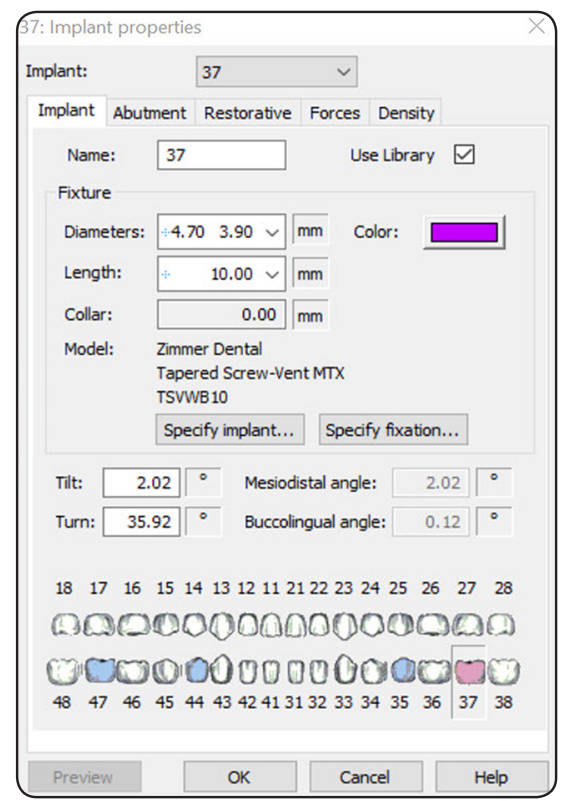

Fig. (6) Bucco-lingual and mesio-distal angles of the real inserted implant at the same site.

With regard to the effect of surgeon experience, there was no significant difference $(p=0.453)$ in the mesio-distal deviation between the expert and novice groups, as the mean deviation for the expert group was $5.6 \pm 4.3$, whereas it was $4.2 \pm 2.94$ for the novice group. In a bucco-lingual direction, there was a significant difference ( $p=0.009$ ) in deviation between the expert and novice surgeons. The expert group performed more accurate implant placement (mean deviation $=3.7 \pm 3.35$ ) in a bucco-lingual 
direction when compared with the novice surgeons (mean deviation $=8.5 \pm 6.3$ ).

With regard to the effect of implant size (Table 1), there was no significant effect of the implant size on the degree of angular deviations either in the mesio-distal ( $p=0.709$ ) or in the bucco-lingual direction $(p=0.734)$. With regard to the interaction effect of experience and implant size on the degree of angular deviation, there was no significant difference between expert regular, expert wide, novice regular, and novice wide groups in both the mesio-distal $(p=0.302)$ and the bucco-lingual direction $(p=0.580)$.

TABLE (1) Shows the mean and standard deviations of different groups.

\begin{tabular}{|c|c|c|c|c|c|}
\hline & Surgeon & Platform & Mean & $\begin{array}{c}\text { Std. } \\
\text { Deviation }\end{array}$ & $\mathrm{N}$ \\
\hline \multirow{9}{*}{ 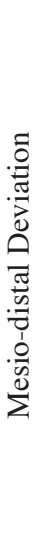 } & \multirow[t]{3}{*}{ Expert } & Regular & 5.9940 & 4.75878 & 15 \\
\hline & & Wide & 5.0930 & 3.76525 & 10 \\
\hline & & Total & 5.6336 & 4.32776 & 25 \\
\hline & \multirow[t]{3}{*}{ Novice } & Regular & 3.5730 & 3.05511 & 10 \\
\hline & & Wide & 5.4800 & 2.50265 & 5 \\
\hline & & Total & 4.2087 & 2.94204 & 15 \\
\hline & \multirow[t]{3}{*}{ Total } & Regular & 5.0256 & 4.26329 & 25 \\
\hline & & Wide & 5.2220 & 3.30742 & 15 \\
\hline & & Total & 5.0993 & 3.88858 & 40 \\
\hline \multirow{9}{*}{ 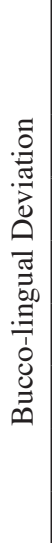 } & \multirow[t]{3}{*}{ Expert } & Regular & 3.6247 & 3.08099 & 15 \\
\hline & & Wide & 3.9790 & 3.90319 & 10 \\
\hline & & Total & 3.7664 & 3.35883 & 25 \\
\hline & \multirow[t]{3}{*}{ Novice } & Regular & 9.0420 & 6.57751 & 10 \\
\hline & & Wide & 7.5660 & 6.47119 & 5 \\
\hline & & Total & 8.5500 & 6.34789 & 15 \\
\hline & \multirow[t]{3}{*}{ Total } & Regular & 5.7916 & 5.39426 & 25 \\
\hline & & Wide & 5.1747 & 4.98217 & 15 \\
\hline & & Total & 5.5602 & 5.18733 & 40 \\
\hline
\end{tabular}

\section{DISCUSSION}

The current study evaluated the influence of surgeon experience on the accuracy of implant placement via a partially guided surgical approach. We found that the expert group performed more accurate implant placement in a bucco-lingual direction when compared with the novice surgeons, which was aligned with our hypothesis.

Multiple in-vitro studies found a statistically significant difference between the experienced and inexperienced surgeons for angular and horizontal error at the implant apex and platform. ${ }^{13},{ }^{14}$ The degree of bucco-lingual deviation in the expert surgeon group in our study was within the normal range of previous studies. The expert surgeon group in Cushen and Turkyilmaz ${ }^{13}$ study performed with a horizontal angular deviation of $2.60 \pm 1.25$. In our study, the expert surgeon group performed with a deviation of $3.7 \pm 3.35$ in the bucco-lingual direction.

The novice surgeon group in Cushen and Turkyilmaz ${ }^{13}$ study performed with an angular deviation of $3.96 \pm 1.64$. It is considered a small amount of deviation if compared with our novice surgeon group, who performed a deviation error of $8.5 \pm 6.3$. The difference between our study and Cushen and Turkyilmaz's study could be due to the clinical environment in both studies. In our study, novice surgeons operated on real patients, whereas in Cushen and Turkyilmaz's study, novice surgeons operated in vitro on a photopolymer resin edentulous mandible.

Cassetta and Bellardini ${ }^{16}$ evaluated the role of surgeon experience in the clinical setting using a mucosa supported surgical guide in completely edentulous patients. The authors found no significant difference between the novice and expert surgeon groups. The amount of angular deviation of the novice surgeon group was 3.07, whereas the angular deviation of the expert surgeon group was 3.21. Such results are different from our 
results due to the characterization of the novice operators in the Cassetta and Bellardini study. The novice operators in their study were five surgeons who have placed approximately 500 implants conventionally without guided surgery, whereas our novice group were surgeons who had no clinical experience in implant placement. However, the authors reported a significant effect of surgeon experience on the accuracy of the position of the surgical guide, indicating a high positioning error for the inexperienced group, which resulted in high apical and coronal horizontal deviation. In our study, all cases involved a teeth support surgical guide to eliminate any systemic error that might result from lack of accurate positioning of the guide during surgery.

Our study is not without limitations. The power of the study could have been increased by involving more patients and, therefore, more implants. However, if more implants were inserted by novice surgeons, it might add to their surgical skills and invite an intra-surgeon variability over time. We invited novice surgeons with no previous experience in implant insertion to mimic the clinical situation of inexperienced undergraduate students or postgraduate trainees at the beginning of their residency program.

Learning the surgical placement of implants is a complex learning task that might impose different types of cognitive load on novice learners, leading to inefficient surgery. According to the cognitive load theory, there are three types of cognitive load, which are intrinsic, extraneous, and germane load. ${ }^{17}$ The intrinsic load results from the complexity of the learning task, which in our case is controlling the drilling speed, retraction of the oral structures, maintaining the mouth opening, regulating the amount of irrigation, holding the handpiece correctly, and drilling in a 3D space while preserving the adjacent vital structures and communicating with the patient. On the other side, the extraneous load refers to the cognitive load that is not necessary for the execution of the learning task. ${ }^{17}$ In implant surgery, such load could emerge from multiple sources such as operator stress, patient anxiety, a fatigued surgeon, and an inappropriate physical environment (e.g., lighting, noise, number of observers in the operating room). Extraneous and/or intrinsic load should be kept to minimum so that cognitive overload is prevented. In other words, the operator would have enough capacity in the working memory, known as germane load, to overcome the other two types of cognitive load, leading to efficient learning.

Generally, intrinsic load can be reduced by performing the simplest form of the whole task then escalating toward a more complex variant of the same task. It was reported that the whole-task approach, where teaching progresses from offering relatively simple, but meaningful, authentic whole tasks to more complex tasks, advocates that performing the whole task is better than dividing it into small separate tasks. ${ }^{18}$ We believe that in the presence of an expert supervisor, using a teeth supported surgical guide in a partially guided surgical protocol could achieve such a goal. It limits the freehand surgery to the last drilling step, which combines the advantages of limiting major deviations from the proposed plan, especially in the bucco-lingual angular direction, and at the same time allows the learner to perform the whole task.

\section{CONCLUSION}

The level of surgeon experience affects the accuracy of implants placed using the teeth supported surgical guide in a bucco-lingual direction; therefore, the use of computer-guided surgery via a partially guided protocol does not completely compensate for the level of operator experience. However, such a surgical guide might be used in a whole-task training of novice surgeons, as it can bridge a gap between simulation training in vitro and freehand surgery in a clinical setting. 


\section{DECLARATION OF INTEREST}

The authors report no declaration of interest in relation to the article.

\section{REFERENCES}

1. Sicilia A, Botticelli D. Computer-guided implant therapy and soft- and hard-tissue aspects. The Third EAO Consensus Conference 2012. Clin Oral Implants Res 2012; 23 Suppl 6: 157-161.

2. Pozzi A, Polizzi G, Moy PK. Guided surgery with toothsupported templates for single missing teeth: A critical review. Eur J Oral Implantol 2016; 9 Suppl 1: S135-153.

3. Marra R, Acocella A, Alessandra R, Ganz SD, Blasi A. Rehabilitation of Full-Mouth Edentulism: Immediate Loading of Implants Inserted With Computer-Guided Flapless Surgery Versus Conventional Dentures: A 5-Year Multicenter Retrospective Analysis and OHIP Questionnaire. Implant Dent 2016.

4. Shen P, Zhao J, Fan L, Qiu H, Xu W, Wang Y, Zhang S, Kim YJ. Accuracy evaluation of computer-designed surgical guide template in oral implantology. J Craniomaxillofac Surg 2015; 43: 2189-2194.

5. D'Haese J, Ackhurst J, Wismeijer D, De Bruyn H, Tahmaseb A. Current state of the art of computer-guided implant surgery. Periodontol 2000 2017; 73: 121-133.

6. Lee KM, Song JM, Cho JH, Hwang HS. Influence of Head Motion on the Accuracy of 3D Reconstruction with ConeBeam CT: Landmark Identification Errors in Maxillofacial Surface Model. PLoS One 2016; 11: e0153210.

7. Baumgaertel S, Palomo JM, Palomo L, Hans MG. Reliability and accuracy of cone-beam computed tomography dental measurements. Am J Orthod Dentofacial Orthop 2009; 136: 19-25; discussion 25-18.

8. Birkfellner W, Solar P, Gahleitner A, Huber K, Kainberger F, Kettenbach J, Homolka P, Diemling M, Watzek G, Bergmann H. In-vitro assessment of a registration protocol for image guided implant dentistry. Clin Oral Implants Res 2001; 12: 69-78.

9. Widmann G, Keiler M, Zangerl A, Stoffner R, Longato $\mathrm{S}$, Bale R, Puelacher W. Computer-assisted surgery in the edentulous jaw based on 3 fixed intraoral reference points. J Oral Maxillofac Surg 2010; 68: 1140-1147.

10. Raico Gallardo YN, da Silva-Olivio IR, Mukai E, Morimoto S, Sesma N, Cordaro L. Accuracy comparison of guided surgery for dental implants according to the tissue of support: a systematic review and meta-analysis. Clin Oral Implants Res 2016.

11. Younes F, Cosyn J, De Bruyckere T, Cleymaet R, Bouckaert E, Eghbali A. A randomized controlled study on the accuracy of free-handed, pilot-drill guided and fully guided implant surgery in partially edentulous patients. J Clin Periodontol 2018; 45: 721-732.

12. Bencharit S, Staffen A, Yeung M, Whitley D, 3rd, Laskin DM, Deeb GR. In Vivo Tooth-Supported Implant Surgical Guides Fabricated With Desktop Stereolithographic Printers: Fully Guided Surgery Is More Accurate Than Partially Guided Surgery. J Oral Maxillofac Surg 2018.

13. Cushen SE, Turkyilmaz I. Impact of operator experience on the accuracy of implant placement with stereolithographic surgical templates: an in vitro study. J Prosthet Dent 2013; 109: 248-254.

14. Hinckfuss S, Conrad HJ, Lin L, Lunos S, Seong WJ. Effect of surgical guide design and surgeon's experience on the accuracy of implant placement. J Oral Implantol 2012; 38 : 311-323.

15. Vermeulen J. The Accuracy of Implant Placement by Experienced Surgeons: Guided vs Freehand Approach in a Simulated Plastic Model. Int J Oral Maxillofac Implants 2016.

16. Cassetta M, Bellardini M. How much does experience in guided implant surgery play a role in accuracy? A randomized controlled pilot study. Int J Oral Maxillofac Surg 2017; 46: 922-930.

17. Young JQ, Van Merrienboer J, Durning S, Ten Cate O. Cognitive Load Theory: implications for medical education: AMEE Guide No. 86. Med Teach 2014; 36: 371-384.

18. Vandewaetere M, Manhaeve D, Aertgeerts B, Clarebout G, Van Merrienboer JJ, Roex A. 4C/ID in medical education: How to design an educational program based on wholetask learning: AMEE Guide No. 93. Med Teach 2015; 37 : 4-20. 